\title{
Comparative Mutagenic Potential of Anti-Influenza Drugs using AMES Salmonella / Microsome mutagenicity Assay
}

\author{
${ }^{1}$ Moneeb Ashraf, ${ }^{2}$ Saadia Shahzad Alam, ${ }^{2}$ Sundas Rafique, ${ }^{3}$ Imran Altaf, ${ }^{4}$ Farooq Sultan \\ ${ }^{1}$ Department of Pharmacology, Postgraduate Medical Institute, Lahore \\ ${ }^{2}$ Department of Pharmacology, FPGMI, Shaikh Zayed Medical Complex, Lahore \\ ${ }^{3}$ Quality Operational Lab, University of Veterinary and Animal Sciences, Lahore \\ ${ }^{4}$ Department of Pharmacology, Gujranwala Medical College, Gujranwala
}

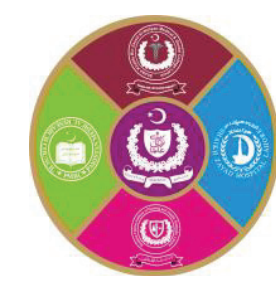

\begin{abstract}
Introduction: Cancer is the second foremost cause of death around the globe. Different drugs have the potential to cause genetic alteration leading to diverse mutations. These mutations play a vigorous role in the etiology of cancers. In modern era, mutagenic potential of drugs can be tested by both in vitro and in vivo studies. AMES mutagenicity assay is one of the initial and rapid in vitro tests to identify the mutagenic potential of different chemicals. Influenza is a respiratory infectious disease caused by the influenza virus. Amantadine, oseltamivir and ribavirin are well known antiviral drugs used for the treatment of influenza with under-researched comparable mutagenic potentials. Aims \& Objectives: To compare mutagenic potentials of amantadine, oseltamivir and ribavirin using AMES Salmonella assay. Place and duration of study: This study was conducted for 2 months at Shaikh Zayed Postgraduate Medical Institute, Lahore and University of Veterinary and Animal Sciences, Lahore. Material \& Methods: Standard plate incorporation and preincubation assays were employed to check the mutagenic potential of fifteen serial two-fold dilutions of amantadine, oseltamivir and ribavirin. These drugs were tested for frame-shift and base pair mutations using histidine-dependent TA-98 and TA-100 strains respectively. The Number of revertant colonies in the test were calculated and compared with negative control to find out the mutagenic index. If any of the mutagenic index value of these tested chemicals was equal to or more than 2 , that drug was considered mutagenic. Results: Comparison produced a significant difference among these materials with p-value 0.001. On pairwise comparison it was observed that amantadine had a significantly lower mutagenic index as compared to ribavirin with p-value 0.003 . Oseltamivir had no significant difference from amantadine and ribavirin with $\mathrm{p}$ values 0.365 and 0.186 respectively. Conclusion: All the tested concentrations of amantadine, oseltamivir and ribavirin are considered non mutagenic because the mutagenic index values for these drugs are below 2 .
\end{abstract}

Key words: Mutagenic potential, Mutagenic index, Salmonella typhi murium

\section{INTRODUCTION}

$\mathrm{D}_{\wedge}$ NA damage may occur as a result of drug treatment for diseases leading to genetic alteration and mutagenicity. These mutations can be point mutation or chromosomal mutations which increase chances of cancer in the patients. ${ }^{1}$ Chromosomal mutations are investigated by sophisticated molecular techniques while point mutations can be easily detected by using bacteria in which mutation changes their growth requirements such as AMES Salmonella assay. ${ }^{2}$ The AMES assay is a revertant mutagenic assay used to establish mutagenic potential of drugs which cause point mutations in histidine dependent Salmonella typhi murium and revert them to histidine independence. ${ }^{3}$

Influenza is a contagious respiratory disease presenting with wide range of symptoms. Standard anti-influenza drugs like amantadine, oseltamivir and ribavirin are used for effective management of the disease. ${ }^{4}$

Amantadine is an adamantine derivative which prevents uncoating and thus replication of the virus. Its use can cause damage to central nervous system and patient can present with nervousness, sleeplessness and aggravation of psychotic symptoms. ${ }^{5}$

Oseltamivir is a neuraminidase inhibitor effective for the management of infections caused by 
influenza A and B virus. Use of this drug can lead to hypersensitivity reactions, liver dysfunction and cardiac \& CNS irregularities. ${ }^{6}$

Ribavirin prevents viral replication by competing with viral RNA nucleosides. It is effective against broad range of RNA viruse. ${ }^{7}$ Its use can cause electrolyte imbalance and cardiac arrhythmias. ${ }^{8}$

Use of anti-influenza drugs is increasing in the modern era because of swine flu and bird flu outbreaks. Due to this reason comparative safety profile of these drugs should be measured. This project was designed to investigate and compare mutagenic potential of amantadine, oseltamivir and ribavirin by AMES Salmonella assay.

\section{MATERIAL AND METHODS}

This study was conducted at the Department of Pharmacology, Shaikh Zayed Postgraduate Medical Institute, Lahore with the co-operation of Quality Operation Laboratory (ISO certified), University of Veterinary and Animal Sciences, Lahore after approval from Institutional Review Board (IRB) of Shaikh Zayed Postgraduate Medical Institute. Stock solutions of amantadine, oseltamivir and ribavirin were prepared by adding $8 \mathrm{mg}$ of drug dissolved in $10 \mathrm{ml}$ of distilled water in separate test tubes to get respective stock solutions having $800 \mu \mathrm{g}$ of the drug per $\mathrm{ml}$. These stock solutions were then diluted two folds till $0.02 \mu \mathrm{g} / \mathrm{ml}$. Mutant strains of Salmonella typhi murium TA 98 and TA 100 were supplied by EBPI Canada in lyophilized form. Genetic confirmation of these strains was done by checking histidine and biotin dependence, rfa marker and pKM 101 plasmid.

Minimal glucose agar was formed by mixing agar, distilled water, Vogel Bonner salt solution and glucose solution $(10 \% \mathrm{v} / \mathrm{v})$. This agar was poured in petri dishes as bottom agar.

\section{Standard plate incorporation assay:}

Top agar was prepared by mixing $2 \mathrm{ml}$ molten agar, $0.50 \mathrm{ml}$ metabolic activation (S-9) mix (EBPI Canada), $0.05 \mathrm{ml}$ of the test chemical dilution and $0.05-0.10 \mathrm{ml}$ overnight culture of the Salmonella strain (about $1-2 \times 10^{8}$ bacteria per tube).

Top agar was poured on bottom agar plates followed by incubation at $37^{\circ} \mathrm{C}$ for 48 hours. Number of histidine revertant colonies were counted and compared with those of negative control for calculating mutagenic index. All the dilutions were tested in triplicate manner.

Sodium azide was used in positive control while distilled water was used in negative control.

\section{Preincubation assay:}

In preincubation assay all the procedure similar to standard plate incorporation method was done with one change that the top agar was incubated at $37^{\circ} \mathrm{C}$ for 20 minutes before pouring it on the bottom agar plates for the formation of active metabolites which themselves can be mutagenic. ${ }^{3}$

\section{Statistical analysis:}

The data was analyzed by statistical package for social Sciences (SPSS IBM statistics 20). For comparing mutagenic potential in terms of mutagenic index, Kruskall Walis ANOVA was used and Mann Whitney U test was applied for post hoc analysis where required. P-value $\leq 0.05$ was considered statistically significant and values between 0.05 and 0.10 were considered indicative.

\section{RESULTS}

\section{Standard plate incorporation assay: \\ TA-100:}

Mutagenic index values for amantadine, oseltamivir and ribavirin using TA-100 at different concentrations are given in Table-1. Mean mutagenic index values for each drug were calculated from values given in the table. These mean mutagenic index values measured for amantadine, oseltamivir and ribavirin were $0.34 \pm 0.13,0.43 \pm 0.15$ and $0.47 \pm 0.15$ respectively. The overall difference among these materials was significant with $\mathrm{p}$-value 0.036 . When pair-wise comparisons were performed none of the difference had $\mathrm{p}$-value $<0.05$, however, amantadine had lower mutagenic index as compared to ribavirin with $\mathrm{p}$ value 0.058 .

\section{TA-98:}

Mutagenic index values for amantadine, oseltamivir and ribavirin using TA-98 at different concentrations is given in Table-2. Mean mutagenic index values for these drugs were calculated from values in the table. Mean mutagenic index recorded for amantadine was smallest with value of $0.40 \pm 0.16$. Oseltamivir had mean mutagenic index value of $0.52 \pm 0.23$ while ribavirin had highest mean mutagenic index value $0.67 \pm 0.23$. The comparison produced significant difference among these materials with p-value 0.001 . On pair wise comparison it was observed that amantadine had significantly lower mutagenic index as compared to ribavirin with p-value 0.003 . Oseltamivir had nonsignificant difference from amantadine and ribavirin with p-values 0.365 and 0.186 respectively. 


\begin{tabular}{|c|c|c|c|c|c|c|c|}
\hline \multirow{4}{*}{ Sr. No. } & \multirow{4}{*}{$\begin{array}{c}\text { Conc. } \\
\mu \mathrm{g} / \\
\text { plate }\end{array}$} & \multicolumn{6}{|c|}{ TA 100} \\
\hline & & \multicolumn{2}{|c|}{ mantadine } & \multicolumn{2}{|c|}{ Oseltamivir } & \multicolumn{2}{|c|}{ Ribavirin } \\
\hline & & M.I. & M.I. & M.I. & M.I. & M.I. & \\
\hline & & & & & & & \\
\hline 1. & 0.02 & 0.278 & \begin{tabular}{|l|l|}
0.134 \\
\end{tabular} & \begin{tabular}{|l|}
0.190 \\
\end{tabular} & 0.164 & \begin{tabular}{|l|}
0.177 \\
\end{tabular} & \begin{tabular}{|l|l|}
0.164 \\
\end{tabular} \\
\hline 2. & 0.04 & 0.291 & 0.164 & \begin{tabular}{|l|}
0.202 \\
\end{tabular} & \begin{tabular}{|l|l|}
0.239 \\
\end{tabular} & \begin{tabular}{|l|l|}
0.215 \\
\end{tabular} & 0.284 \\
\hline 3. & 0.09 & 0.316 & \begin{tabular}{|l|l|}
0.179 \\
\end{tabular} & \begin{tabular}{|l|}
0.240 \\
\end{tabular} & 0.254 & \begin{tabular}{|l|l|}
0.278 \\
\end{tabular} & 0.298 \\
\hline 4. & 0.19 & 0.367 & 0.253 & 0.266 & 0.313 & \begin{tabular}{|l|l|}
0.354 \\
\end{tabular} & 0.343 \\
\hline 5. & 0.39 & 0.405 & \begin{tabular}{|l|l|} 
\\
\end{tabular} & 0.304 & 0.328 & \begin{tabular}{|l|l|}
0.380 \\
\end{tabular} & 0.388 \\
\hline 6. & 0.78 & 0.456 & \begin{tabular}{|l|l|} 
\\
\end{tabular} & 0.367 & \begin{tabular}{|l|l|} 
\\
\end{tabular} & \begin{tabular}{|l|}
0.392 \\
\end{tabular} & \begin{tabular}{|l|l|} 
\\
\end{tabular} \\
\hline 7. & 1.56 & 0.468 & \begin{tabular}{|l|l|}
0.388 \\
\end{tabular} & \begin{tabular}{|l|}
0.380 \\
\end{tabular} & \begin{tabular}{|l|l|}
0.448 \\
\end{tabular} & \begin{tabular}{|l|l|}
0.456 \\
\end{tabular} & \begin{tabular}{|l|}
0.478 \\
\end{tabular} \\
\hline 8. & 3.125 & 0.468 & \begin{tabular}{|l|l|}
0.433 \\
\end{tabular} & \begin{tabular}{|l|}
0.405 \\
\end{tabular} & \begin{tabular}{|l|l|}
0.463 \\
\end{tabular} & \begin{tabular}{|l|l|}
0.481 \\
\end{tabular} & \begin{tabular}{|l|l|}
0.537 \\
\end{tabular} \\
\hline 9. & 6.25 & 0.519 & \begin{tabular}{|l|l|}
0.478 \\
\end{tabular} & \begin{tabular}{|l|} 
\\
\end{tabular} & \begin{tabular}{|l|l|}
0.492 \\
\end{tabular} & \begin{tabular}{|l|}
0.544 \\
\end{tabular} & 0.582 \\
\hline 10. & 12.5 & 0.557 & 0.567 & 0.481 & 0.552 & \begin{tabular}{|l|}
0.569 \\
\end{tabular} & \begin{tabular}{|l|l|}
0.597 \\
\end{tabular} \\
\hline 11. & 25 & 0.607 & \begin{tabular}{|l|l|} 
& 0.463 \\
\end{tabular} & \begin{tabular}{|l|}
0.544 \\
\end{tabular} & \begin{tabular}{|l|l|}
0.657 \\
\end{tabular} & \begin{tabular}{|l|}
0.620 \\
\end{tabular} & 0.657 \\
\hline 12. & 50 & 0.494 & 0.448 & 0.595 & \begin{tabular}{|l|}
0.642 \\
\end{tabular} & \begin{tabular}{|l|}
0.709 \\
\end{tabular} & 0.701 \\
\hline 13. & 100 & 0.456 & 0.418 & 0.557 & \begin{tabular}{|l|l|} 
& 0.597 \\
\end{tabular} & \begin{tabular}{|l|}
0.671 \\
\end{tabular} & 0.612 \\
\hline 14. & 200 & 0.380 & 0.373 & 0.456 & 0.492 & \begin{tabular}{|l|}
0.392 \\
\end{tabular} & 0.522 \\
\hline 15. & 400 & 0.354 & 0.284 & $0.367 \mid$ & \begin{tabular}{|l|l|} 
\\
\end{tabular} & \begin{tabular}{|l|}
0.380 \\
\end{tabular} & 0.478 \\
\hline+ +Control & 5 & 14.094 & 12.883 & 14.094 & 12.883 & \begin{tabular}{|l|}
14.094 \\
\end{tabular} & 12.883 \\
\hline
\end{tabular}

Table-1: Mutagenic Index values of increasing doses of Amantadine, oseltamivir and ribavirin using TA-100 in the presence and absence of S9 metabolic extract

\begin{tabular}{|c|c|c|c|c|c|c|c|}
\hline \multirow{4}{*}{ Sr. No. } & \multirow{4}{*}{$\begin{array}{c}\text { Conc. } \\
\mu \mathrm{g} / \\
\text { plate }\end{array}$} & \multicolumn{6}{|c|}{ TA 98} \\
\hline & & \multicolumn{2}{|c|}{ Amantadine } & \multicolumn{2}{|c|}{ Oseltamivir } & \multicolumn{2}{|c|}{ Ribavirin } \\
\hline & & M.I. & M.I. & M.I. & M.I. & M.I. & M.I. \\
\hline & & $+\mathrm{S}-9$ & -S-9 & $+\mathrm{S}-9$ & -S-9 & $+\mathrm{S}-9$ & -S-9 \\
\hline 1. & 0.02 & 0.255 & \begin{tabular}{|l|}
0.163 \\
\end{tabular} & \begin{tabular}{|l|}
0.157 \\
\end{tabular} & \begin{tabular}{|l|}
0.093 \\
\end{tabular} & 0.235 & \begin{tabular}{|l|}
0.209 \\
\end{tabular} \\
\hline 2. & 0.04 & 0.255 & \begin{tabular}{|l|}
0.209 \\
\end{tabular} & \begin{tabular}{|l|l|}
0.196 \\
\end{tabular} & \begin{tabular}{|l|}
0.209 \\
\end{tabular} & 0.294 & \begin{tabular}{|l|}
0.372 \\
\end{tabular} \\
\hline 3. & 0.09 & 0.314 & 0.233 & \begin{tabular}{|l|}
0.255 \\
\end{tabular} & 0.256 & \begin{tabular}{|l|}
0.333 \\
\end{tabular} & \begin{tabular}{|l|l|}
0.419 \\
\end{tabular} \\
\hline 4. & 0.19 & 0.353 & 0.256 & \begin{tabular}{|l|l|}
0.294 \\
\end{tabular} & 0.302 & 0.392 & 0.465 \\
\hline 5. & 0.39 & 0.372 & 0.279 & \begin{tabular}{|l|}
0.372 \\
\end{tabular} & 0.419 & 0.451 & 0.488 \\
\hline 6. & 0.78 & 0.412 & 0.395 & \begin{tabular}{|l|}
0.392 \\
\end{tabular} & 0.512 & 0.529 & 0.628 \\
\hline 7. & 1.56 & 0.471 & 0.488 & 0.470 & 0.581 & 0.608 & \begin{tabular}{|l|}
0.721 \\
\end{tabular} \\
\hline 8. & 3.125 & 0.529 & 0.488 & \begin{tabular}{|l|}
0.549 \\
\end{tabular} & 0.651 & 0.647 & 0.767 \\
\hline 9. & 6.25 & 0.569 & 0.535 & \begin{tabular}{|l|l|}
0.608 \\
\end{tabular} & 0.698 & 0.706 & 0.907 \\
\hline 10. & 12.5 & 0.627 & 0.628 & \begin{tabular}{|l|}
0.627 \\
\end{tabular} & 0.721 & 0.784 & 0.907 \\
\hline 11. & 25 & 0.725 & \begin{tabular}{|l|l|} 
\\
\end{tabular} & \begin{tabular}{|l|} 
\\
\end{tabular} & 0.837 & \begin{tabular}{|l|l|}
0.823 \\
\end{tabular} & $\mid 0.930$ \\
\hline 12. & 50 & 0.588 & 0.558 & \begin{tabular}{|l|}
0.784 \\
\end{tabular} & 0.860 & 0.941 & 0.953 \\
\hline 13. & 100 & 0.529 & 0.465 & \begin{tabular}{|l|} 
\\
\end{tabular} & 0.698 & 0.863 & 0.884 \\
\hline 14. & 200 & 0.412 & 0.395 & \begin{tabular}{|l|}
0.569 \\
\end{tabular} & \begin{tabular}{|l|l|}
0.581 \\
\end{tabular} & $\mid 0.725$ & \begin{tabular}{|l|l|}
0.767 \\
\end{tabular} \\
\hline 15. & 400 & 0.314 & \begin{tabular}{|l|l|}
0.279 \\
\end{tabular} & \begin{tabular}{|l|}
0.451 \\
\end{tabular} & 0.442 & \begin{tabular}{|l|}
0.569 \\
\end{tabular} & \begin{tabular}{|l|}
0.698 \\
\end{tabular} \\
\hline+ Control & 5 & 10.502 & 9.928 & 10.502 & \begin{tabular}{|l|}
9.928 \\
\end{tabular} & 10.502 & 9.928 \\
\hline
\end{tabular}

Table-2: Mutagenic Index values of increasing doses of Amantadine, oseltamivir and ribavirin using TA-98 in the presence and absence of S9 metabolic extract

\section{Preincubation assay:}

\section{TA-100:}

Mutagenic index values for these drugs is given in Table-1. Means of these values are calculated. Mean mutagenic index values for amantadine, oseltamivir and ribavirin were $0.43 \pm 0.10,0.38 \pm 0.13$ and $0.44 \pm 0.16$ respectively. The difference was nonsignificant with p-value 0.099 .

\section{TA-98:}

Mutagenic index values for these drugs are given in Table-2. Mean of these values was calculated. Mean mutagenic index values for amantadine, oseltamivir and ribavirin were $0.45 \pm 0.14,0.47 \pm 0.19$ and $0.59 \pm 0.22$ respectively. The difference was not significant with p-value 0.069 .

\section{DISCUSSION}

Mutation is irreversible damage to the genome which can be because of alteration in either one of the base pairs or a chromosome. These mutations change cellular programing resulting in uncontrolled growth of cells. AMES test is one of the bacterial assays used for testing base pair and frame shift mutations. These mutations revert histidine dependence of Salmonella typhi murium strains. ${ }^{3}$ Carbon tetrachloride was reported mutagenic using TA-98 strain in the absence of rat liver extract S9 while chloroform showed mutagenicity with WP2/ PKM101 strain in the presence of glutathione supplemented S9 extract. ${ }^{9}$ In another study two stimulated atmospheres having different proportions of pollutants were tested positive for base pair mutations using TA-100 and TA-104 strains. $^{10}$ Abused drug ecstasy showed no reversion in histidine dependent bacteria. ${ }^{11}$ In a study, ecofriendly protic ionic fluids were reported negative for mutagenic potential through mini Ames test. ${ }^{12}$ Pulpotomy agents are frequently used in dental procedures and their genotoxicity was checked with the results that ferric sulphate and formocresol came out to be mutagenic while CEM cement was non mutagenic. ${ }^{13}$

This project was designed to compare mutagenic potential of amantadine, oseltamivir and ribavirin assessed by AMES Salmonella assay. Use of these standard anti influenza drugs is increasing with the increased epidemics of bird and swine flu.

Amantadine is effective against influenza A virus but many subtypes of this virus have developed resistance against amantadine. ${ }^{14}$ In a case report high dose of amantadine (1000ng/ml) led to encephalopathy in patient with normal renal function. ${ }^{15}$ In a study on adult male CF mice amantadine caused no bone marrow suppression or DNA damage at low doses tested by micronucleus assay. But on high doses it caused damage to DNA of the brain and severe neurotoxicity. ${ }^{16}$ 
Oseltamivir is effective against both influenza A and $\mathrm{B}$ viruses by preventing shedding of virions. In recent epidemics there is reported increase in resistance against this drug because of mutations in neuraminidase. ${ }^{17}$ In a study on cultured lymphocytes, oseltamivir was reported non genotoxic with no significant increase in chromosomal aberration and micro nucleated cells. ${ }^{18}$ In another study oseltamivir reduced cisplatin and gemcitabine resistance to pancreatic cancer by targeting neu 1 sialidase. ${ }^{19}$

Ribavirin blocks RNA synthesis which makes it effective against influenza virus. Due to increased resistance against amantadine and oseltamivir, use of ribavirin is increasing to decrease disease duration and complications. Micronucleus assay was done to check genotoxic effect of ribavirin on rat bone marrow which led to increase in the incidence of micronuclei. ${ }^{20}$ In another study it was proposed that ribavirin causes chromosomal damage leading to production of fragments for micronuclei. ${ }^{21}$ In a study on wistar rats it was observed that ribavirin induced morphological changes in the shape of head and tail of sperm. ${ }^{22}$

Our study reports no significant mutagenic potential of amantadine, oseltamivir and ribavirin by AMES Salmonella assay as all the mutagenic index values were below 2 with either of the strain (TA-98 or TA-100) with or without S9 as given in Table-1 and Table-2. These results are similar to the previous studies which also reported no mutagenic potential of these drugs by AMES test. On pair wise comparison between these drugs it was observed that amantadine had significantly lower mutagenic index as compared to ribavirin with p-value 0.003 while oseltamivir had no significant difference from amantadine and ribavirin with p-values 0.365 and 0.186 respectively. Limitations of the study is that AMES test only identifies point mutations and don't check chromosomal mutations so it is recommended that comparative study of chromosomal mutations of these drugs should also be done.

\section{CONCLUSION}

We therefore conclude that amantadine, oseltamivir and ribavirin are non-mutagenic by AMES Salmonella assay with ribavirin having highest mutagenic index values among them. Although these drugs came out to be non-mutagenic by this assay, still ribavirin needs further comparative mutagenic potential studies based on its literature review as this assay identifies only base pair and frame shift mutations induced by the drugs.

\section{REFERENCES}

1. Chen L, Liu P, Evans TC, Ettwiller LM. DNA damage is a pervasive cause of sequencing errors, directly confounding variant identification. Science. 2017; 355(6326):752-6.

2. Gatehouse D, Haworth S, Cebula T, Gocke E, Kier L, Matsushima T, et al. Recommendations for the performance of bacterial mutation assays. Mutation Research/ Environmental Mutagenesis and Related Subjects. 1994; 312 (3):217-33.

3. Mortelmans K, Zeiger E. The Ames Salmonella/microsome mutagenicity assay. Mutation Research/Fundamental and Molecular Mechanisms of Mutagenesis.2000;455(1):29-60.

4. Stiver G. The treatment of influenza with antiviral drugs. Canadian Medical Association Journal. 2003; 168(1):49-57.

5. Keyser LA, Karl M, Nafziger AN, Bertino JS. Comparison of central nervous system adverse effects of amantadine and rimantadine used as sequential prophylaxis of influenza A in elderly nursing home patients. Archives of internal medicine. 2000; 160(10):1485-8.

6. Strong M, Burrows J, Stedman E, Redgrave P. Adverse drug effects following oseltamivir mass treatment and prophylaxis in a school outbreak of 2009 pandemic influenza A (H1N1) in June 2009, Sheffield, United Kingdom. Euro Surveill. 2010; 15(19):19565-70.

7. Markland W, McQuaid T, Jain J, Kwong A. Broad-spectrum antiviral activity of the IMP dehydrogenase inhibitor VX-497: a comparison with ribavirin and demonstration of antiviral additivity with alpha interferon. Antimicrobial agents and chemotherapy. 2000; 44(4):859-66.

8. Chiou H-E, Liu C-L, Buttrey MJ, Kuo H-P, Liu $\mathrm{H}-\mathrm{W}$, Kuo H-T, et al. Adverse effects of ribavirin and outcome in severe acute respiratory syndrome: experience in two medical centers. CHEST Journal. 2005; 128(1): 263-72.

9. Araki A, Kamigaito N, Sasaki T, Matsushima T. Mutagenicity of carbon tetrachloride and chloroform in Salmonella typhimurium TA98, TA100, TA1535, and TA1537, and Escherichia coli WP2uvrA/pKM101 and WP2/pKM101, using a gas exposure method. Environmental and molecular mutagenesis. 2004; 43(2):128-33.

10. Zavala J, Krug JD, Warren SH, Krantz QT, King C, McKee J, et al. Evaluation of an air quality health index for predicting the mutagenicity of simulated atmospheres. 
Environmental science \& technology. 2018; 52(5):3045-53.

11. Jalali A, Hariri M, Farajzadeh A, Khajeamiri E. Assessing Mutagenicity of the drug Mdma (Ecstasy) available in Iran using Ames Bioassay. Jundishapur Journal of Natural Pharmaceutical Products. 2010; 5(1):44-9.

12. Reid JE, Sullivan N, Swift L, Hembury GA, Shimizu S, Walker AJ. Assessing the mutagenicity of protic ionic liquids using the mini Ames test. Sustainable Chemical Processes. 2015; 3(1):17.

13. Samiei M, Asgary S, Farajzadeh M, Bargahi N, Abdolrahimi M, Kananizadeh $U$, et al. Investigating the mutagenic effects of three commonly used pulpotomy agents using the ames test. Advanced pharmaceutical bulletin. 2015;5(1):121.

14. Tosh C, Murugkar H, Nagarajan S, Tripathi S, Katare M, Jain R, et al. Emergence of amantadine-resistant avian influenza H5N1 virus in India. Virus genes. 2011;42(1):10-5.

15. Albrektson K, Mandviwala A, Donatelli C, Soliman D, Lackamp J, Teba C. Amantadine Toxicity: Uncovering a Rare Cause of Severe Encephalopathy. D46 Critical Care Case Reports: Toxicology And Poisonings: American Thoracic Society; 2018. p. A6912-A.

16. Kaefer V, Semedo JG, Kahl S, Francília V, Borowsky V, Gomes R, et al. DNA damage in brain cells and behavioral deficits in mice after treatment with high doses of amantadine.Journal of applied toxicology. 2010; 30(8):745-53.

17. Baz M, Abed Y, Simon P, Hamelin M-È, Boivin G. Effect of the neuraminidase mutation $\mathrm{H} 274 \mathrm{Y}$ conferring resistance to oseltamivir on the replicative capacity and virulence of old and recent human influenza A (H1N1) viruses. The Journal of infectious diseases.2010;201(5):740-5.

18. Ila HB, Ilhan A. In vitro genotoxic perspective of Tamiflu. Cytotechnology. 2012;64(4):443-9.

19. O'Shea LK, Abdulkhalek S, Allison S, Neufeld RJ, Szewczuk MR. Therapeutic targeting of Neu1 sialidase with oseltamivir phosphate (Tamiflu $\left.{ }^{\circledR}\right)$ disables cancer cell survival in human pancreatic cancer with acquired chemoresistance. OncoTargets and therapy. 2014; 7:117.
20. Narayana K, D'Souza UJ, Rao KS. The genotoxic and cytotoxic effects of ribavirin in rat bone marrow. Mutation Research/Genetic Toxicology and Environmental Mutagenesis. 2002; 521(1):179-85.

21. Rao KS, Narayana K. In vivo chromosome damaging effects of an inosine monophosphate dehydrogenase inhibitor: Ribavirin in mice. Indian journal of pharmacology. 2005;37(2):90.

22. Narayana K, D'Souza UJ, Rao KS. Ribavirininduced sperm shape abnormalities in Wistar rat. Mutation Research/Genetic Toxicology and Environmental Mutagenesis.2002;513(1):193-6.

\section{The Authors:}

Dr. Moneeb Ashraf,

Assistant Prof.

Department of Pharmacology,

Postgraduate Medical Institute, Lahore

Prof. Saadia Shahzad Alam,

Head, of Pharmacology Department,

PGMI, Shaikh Zayed Medical Complex, Lahore

Dr. Sundas Rafique,

House Officer,

Department of Pharmacology,

PGMI, Shaikh Zayed Medical Complex, Lahore

Dr. Imran Altaf,

Associate Prof.

Quality Operational Lab,

University of Veterinary and Animal Sciences,

Lahore.

Dr. Farooq Sultan,

Demonstrator,

Department of Pharmacology,

Gujranwala Medical College, Gujranwala

\section{Corresponding Author:}

Dr. Moneeb Ashraf,

Assistant Prof.

Department of Pharmacology,

Postgraduate Medical Institute, Lahore

Email: Moneeb-ashraf@hotmail.com 